\title{
Rate-Constrained Collaborative Noise Reduction for Wireless Hearing Aids
}

\author{
Olivier Roy, Student Member, IEEE, and Martin Vetterli, Fellow, IEEE
}

\begin{abstract}
Hearing aids are electronic, battery-operated sensing devices which aim at compensating various kinds of hearing impairments. Recent advances in low-power electronics coupled with progresses made in digital signal processing offer the potential for substantial improvements over state-of-the-art systems. Nevertheless, efficient noise reduction in complex listening scenarios remains a challenging task, partly due to the limited number of microphones that can be integrated on such devices. We investigate the noise reduction capability of hearing instruments that may exchange data by means of a rate-constrained wireless link and thus benefit from the signals recorded at both ears of the user. We provide the necessary theoretical results to analyze this collaboration mechanism under two different coding strategies. The first approach takes full benefit of the binaural correlation, while the second neglects it, since binaural statistics are difficult to estimate in a practical setting. The gain achieved by collaborating hearing aids as a function of the communication bit rate is then characterized, both in a monaural and a binaural configuration. The corresponding optimal rate allocation strategies are computed in closed form. While the analytical derivation is limited to a simple acoustic scenario, the latter is shown to capture many of the features of the general problem. In particular, it is observed that the loss incurred by coding schemes which do not consider the binaural correlation is rather negligible in a very noisy environment. Finally, numerical results obtained using real measurements corroborate the potential of our approach in a realistic scenario.
\end{abstract}

Index Terms-Beamforming, binaural noise reduction, collaborating hearing aids, remote source coding with side information, spatial filtering, wireless link.

\section{INTRODUCTION}

W E consider the binaural hearing aid configuration illustrated in Fig. 1. Two hearing aids, each equipped with a set of microphones, a computing unit and wireless commu-

Manuscript received December 21, 2006; revised October 11, 2008. First published November 11, 2008; current version published January 30, 2009. The associate editor coordinating the review of this manuscript and approving it for publication was Venkatesh Saligrama. This work was supported by the National Competence Center in Research on Mobile Information and Communication Systems (NCCR-MICS, http://www.mics.org), a center supported by the Swiss National Science Foundation under Grant 5005-67322. The material in this paper was presented in part at the MSRI Workshop on Mathematics of Relaying and Cooperation in Communication Networks, Berkeley, CA, April 2006, and at the IEEE International Symposium on Information Theory, Seattle, WA, July 2006.

O. Roy is with the School of Computer and Communication Sciences, Ecole Polytechnique Fédérale de Lausanne, 1015 Lausanne, Switzerland (e-mail: olivier.roy@epfl.ch).

M. Vetterli is also with the Department of Electrical Engineering and Computer Sciences, University of California, Berkeley, CA 94720 USA (e-mail: martin.vetterli@epfl.ch).

Color versions of one or more of the figures in this paper are available online at http://ieeexplore.ieee.org.

Digital Object Identifier 10.1109/TSP.2008.2009267 nication capabilities, are mounted on the left and right ear of a user. They record the acoustic field induced by the presence of multiple sound sources and exchange their acquired signals by means of a wireless communication link. The ultimate goal is to provide the loudspeakers with signals that overcomes the user's hearing impairments. To this end, each instrument coherently combines its own signals and those transmitted by the contralateral device in order to extract a desired sound source coming from a particular direction. This process is commonly referred to as beamforming and has been extensively studied over the past decades in the array processing literature [1], [2].

The situation is different here in the sense that we specifically take into account the rate-constrained nature of the communication link connecting the two hearing aids. Under this assumption, the beamforming paradigm corresponds to what is known, in the information theory literature, as a (remote) multiterminal source coding (or rate-distortion) problem [3]. From the perspective of one hearing device, the task reduces to a source coding problem with side information at the decoder, where the source is merely observed in a remote fashion. It is worth pointing out that an equivalent configuration arises when the hearing aid is wirelessly connected to a generic assistive listening device (e.g., a lecturer's microphone). The forthcoming analysis hence also applies in this scenario. The goal here is to characterize the optimal tradeoff between the communication rate sustainable by the wireless link and the reconstruction accuracy of the desired source. This problem, referred to as remote, indirect or noisy Wyner-Ziv coding in the literature, has been addressed by various researchers in the scalar case [3]-[5]. More generally, Witsenhausen [6] elegantly demonstrated how certain classes of remote rate-distortion problems can be reduced to direct ones, unifying earlier results by Dobrushin and Tsybakov [7], Sakrison [8] and Wolf and Ziv [9]. Extension to vector sources was investigated by Rebollo-Monedero et al. in the context of high-rate transform coding [10], [11]. In order to cope with the problem at hand, we provide an evaluation of these results to jointly Gaussian stationary random processes. The distortion measure considered here is the weighted mean-squared error (MSE) criterion. Because the optimal approach requires statistics which are difficult to estimate in a practical setting, we also derive the rate-distortion tradeoff obtained when the presence of side information is neglected by the encoder.

The idea to investigate collaboration between hearing aids primary stems from the fact that, limited by obvious design considerations, a microphone array embedded on one hearing device remains of small spatial extent. This restriction will be even more apparent in the future as miniaturization plays an important role in the acceptance of such devices by hearing im- 
paired people. Establishing a link between the two hearing instruments allows us to benefit from the distance between the two ears and thus permits better beamforming resolution [1]. It is worth noting that the aforementioned limitation has fostered a substantial body of research focusing on the design of larger microphone arrays [12], possibly mounted on glasses [13] or in the shape of a necklace [14]. Other works have considered, as we do here, the array formed by connecting microphones available at both hearing aids [15]-[19]. Moreover, these topics have been the subject of various industrial patents [20]-[24], highlighting the practical relevance of the problem. Recently, Doclo et al. [25] investigated the use of dimensionality reduction schemes to address the bandwidth limitation of the wireless link. The rate-constrained nature of the communication medium has however, to the best of our knowledge, never been taken into account in previous studies.

The analysis presented in this paper is of information-theoretic nature. It thus ignores the stringent delay and processing complexity constraints inherent to the considered hearing aid problem. Typically, a digital hearing aid must operate with a power supply no greater than $1 \mathrm{~V}$ and delays introduced by coding and wireless transmission cannot exceed a few milliseconds. The results presented in this work, however, serve as useful upper bounds to the gain achievable by practical schemes, and provide insights on how to optimally allocate the available communication resources. To this end, the problem is first studied from the perspective of one hearing aid. We consider a simple acoustic scenario for which we can derive analytical formulas of the beamforming gain as a function of the communication bit rate for two different coding strategies. We then look at the system globally, that is with bidirectional communications between the hearing instruments. For the simple scenario considered previously, similar analytical gain-rate tradeoffs are derived and optimal rate allocation policies are presented. Finally, various features of the considered binaural hearing aid system are illustrated using real data measured in a realistic reverberant environment.

The paper is organized as follows. In Section II, we state the problem of binaural noise reduction. The necessary information-theoretical results are derived in Section III. Monaural gain-rate tradeoffs are studied in Section IV in a simple acoustic scenario. A similar analysis is then presented in the binaural case in Section V, along with optimal rate allocation strategies. Finally, Section VI provides numerical results obtained using real measurements, and Section VII concludes the paper.

\section{BINAURAL HEARING AIDS}

Let us consider the binaural configuration schematically depicted in Fig. 1(a). A user carries a left and a right hearing aid, indexed with 1 and 2, respectively. They both comprise a set of microphones, a processing unit with wireless communication capabilities and a loudspeaker. The signal recorded by the $M_{1}$ microphones of hearing aid 1 can be expressed as

$$
X_{1, m}(t)=S_{1, m}(t)+N_{1, m}(t) \text { for } m=1,2, \ldots, M_{1}
$$

where $S_{1, m}(t)$ denotes the speech (desired) component and $N_{1, m}(t)$ the noise (undesired) component. Similarly, the signals

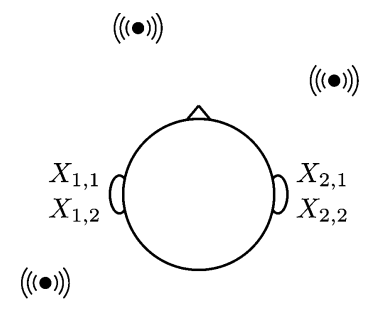

$((\bullet \bullet))$

(a)

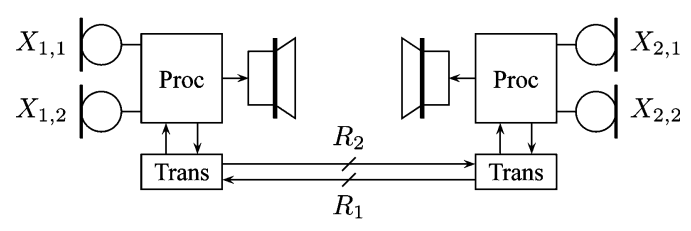

(b)

Fig. 1. Binaural hearing aids. (a) Typical recording setup. (b) Collaboration using rate-constrained wireless communication links.

recorded at the $M_{2}$ microphones of hearing aid 2 can be decomposed as $X_{2, m}(t)=S_{2, m}(t)+N_{2, m}(t)$ for $m=1,2, \ldots, M_{2}$. Note that, in the sequel, the variable $t$ is omitted for conciseness. For convenience, we will write the input signals in vector form as

$$
\mathbf{X}_{l}=\left[X_{l, 1}, X_{l, 2}, \ldots, X_{l, M_{l}}\right]^{T} \text { for } l=1,2
$$

where $\mathbf{X}_{l}=\mathbf{S}_{l}+\mathbf{N}_{l}$ with $\mathbf{S}_{l}$ and $\mathbf{N}_{l}$ defined similarly as $\mathbf{X}_{l}$. The speech vector $\mathbf{S}_{l}$ and the noise vector $\mathbf{N}_{l}$ are modeled as independent zero-mean jointly Gaussian stationary random vector processes. Let us denote by $\Phi_{S_{l}}^{\left(m, m^{\prime}\right)}(\Omega)$ the (cross) power spectral density (PSD) between $S_{l, m}$ and $S_{l, m^{\prime}}$, and by $\Phi_{N_{l}}^{\left(m, m^{\prime}\right)}(\Omega)$ that between $N_{l, m}$ and $N_{l, m^{\prime}}$. To lighten the notation, the variable $\Omega$ will be also omitted in the rest of the discussion. The $M_{l} \times M_{l}$-dimensional PSD matrix of the speech and noise component can thus be expressed as

$$
\begin{gathered}
\boldsymbol{\Phi}_{S_{l}}=\left[\begin{array}{cccc}
\Phi_{S_{l}}^{(1,1)} & \Phi_{S_{l}}^{(1,2)} & \ldots & \Phi_{S_{l}}^{\left(1, M_{l}\right)} \\
\Phi_{S_{l}}^{(2,1)} & \Phi_{S_{l}}^{(2,2)} & \ldots & \Phi_{S_{l}}^{\left(2, M_{l}\right)} \\
\vdots & \vdots & \ddots & \vdots \\
\Phi_{S_{l}}^{\left(M_{l}, 1\right)} & \Phi_{S_{l}}^{\left(M_{l}, 2\right)} & \ldots & \Phi_{S_{l}}^{\left(M_{l}, M_{l}\right)}
\end{array}\right] \\
\boldsymbol{\Phi}_{N_{l}}=\left[\begin{array}{cccc}
\Phi_{N_{l}}^{(1,1)} & \Phi_{N_{l}}^{(1,2)} & \ldots & \Phi_{N_{l}}^{\left(1, M_{l}\right)} \\
\Phi_{N_{l}}^{(2,1)} & \Phi_{N_{l}}^{(2,2)} & \ldots & \Phi_{N_{l}}^{\left(2, M_{l}\right)} \\
\vdots & \vdots & \ddots & \vdots \\
\Phi_{N_{l}}^{\left(M_{l}, 1\right)} & \Phi_{N_{l}}^{\left(M_{l}, 2\right)} & \ldots & \Phi_{N_{l}}^{\left(M_{l}, M_{l}\right)}
\end{array}\right]
\end{gathered}
$$

respectively. The PSD matrix of the vector process $\mathbf{X}_{l}$ then follows as $\boldsymbol{\Phi}_{X_{l}}=\boldsymbol{\Phi}_{S_{l}}+\boldsymbol{\Phi}_{N_{l}}$. Note that here, the statistical characteristics of the involved sources are assumed to be known at both hearing aids.

The goal of each hearing instrument is to recover the speech component of one of its microphones (say microphone 1) with minimum distortion. To make matters clearer, let us adopt the perspective of hearing aid 1. Its goal is to estimate the signal $S_{1,1}$ with minimum weighted MSE based on its own observations $\mathbf{X}_{1}$ and a compressed version of the signals $\mathbf{X}_{2}$ recorded 


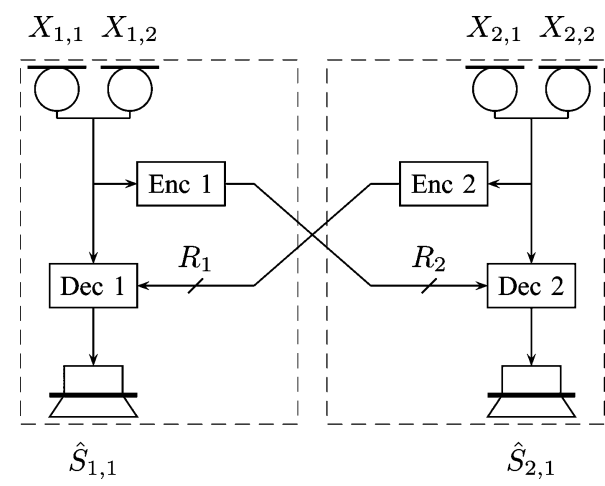

Fig. 2. Binaural hearing aids with $M_{1}=M_{2}=2$ microphones.

at the microphones of hearing aid 2. The choice of a minimum weighted MSE criterion is partly motivated by the fact that the optimality of the MSE processor extends to various other criteria with minor modifications [2]. While some of the characteristics of the human ear can be taken into account by means of an appropriate weighting, it is important to point out that the weighted MSE does not take into consideration other important factors related to speech intelligibility in noise. More relevant speech intelligibility measures can be found in the literature (see, e.g., [26]), but are usually not amenable to closed-form optimization. More generally, an accurate distortion measure seems rather elusive owing to the complexity of human hearing. In this context, the MSE criterion is appealing in that it captures the general features of speech in noise while being simple enough to derive optimal processing strategies.

With the above considerations, the key is to realize that our setup simply corresponds to a remote source coding problem with side information and a weighted MSE criterion [4]. More specifically, we wish to encode the vector signal $\mathbf{X}_{2}$ with rate $R_{1}$ such as to minimize the distortion between $S_{1,1}$ and its reconstruction $\hat{S}_{1,1}$, with the side information $\mathbf{X}_{1}$ available (only) at the decoder. This procedure is illustrated for both devices in Fig. 2. The monaural gain achieved at hearing aid $l$ is defined as

$$
G_{l}\left(R_{l}\right)=\frac{D_{l}(0)}{D_{l}\left(R_{l}\right)} \text { for } l=1,2
$$

where $D_{l}\left(R_{l}\right)$ denotes the corresponding optimal rate-distortion tradeoff, ${ }^{1} D_{l}(0)$ being the distortion incurred when there is no collaboration $\left(R_{l}=0\right)$. Note that $R_{l}$ refers to the rate at which data is delivered to hearing aid $l$. The quantity $G_{l}\left(R_{l}\right)$ actually corresponds to the signal-to-distortion improvement enabled by the wireless link when it operates at rate $R_{l}$. The binaural gain provided by the wireless collaboration mechanism can thus be obtained as

$$
G(R)=\frac{D(0)}{D(R)}
$$

where $D(R)$ refers to the optimal tradeoff between the sum distortion $D=D_{1}+D_{2}$ and the total (bidirectional) transmission rate $R=R_{1}+R_{2}$. In this binaural configuration, the chosen distortion criterion appears to be the most natural one considering

\footnotetext{
${ }^{1}$ In this discussion, $R(D)$ and $D(R)$ will both be referred to as optimal rate-
} distortion tradeoffs. the inherent symmetry of the human hearing system. Different weighting operators may be used at the left and right ear to take into account unequal characteristics. As mentioned previously, a weighted MSE criterion only partially reflects the properties of binaural hearing, a topic which is currently matter of great research efforts (see, e.g., [27]). In particular, the MSE criterion preserves the spatial characteristics of the speech source but modifies those of the noise component [28]. The overall auditory image may thus be modified by the noise reduction scheme. This may be circumvented, for example, by adding a small fraction of the original binaural signal to the output [29]. The MSE criterion nevertheless provides a useful means to design noise reduction algorithms and is often considered in practice. Let us now present the results needed to compute the above gain-rate functions under our Gaussian assumptions.

\section{Remote Source Coding With Side Information}

This section exposes the information-theoretical results needed to analyze the problem of binaural hearing aids described previously. In Section III-A, we first look at the achievable rate-distortion region for the remote source coding problem with side information for jointly Gaussian stationary random vector processes. While being optimal in a rate-distortion sense, side information aware (SIA) transmission protocols resort to coding techniques, described for example in [30], which require the knowledge of statistics that are difficult to estimate in practice owing to the distributed nature of the communication setup. With this restriction in mind, Section III-B considers the scenario where the data is encoded in a rate-distortion optimal fashion for a decoder that does not have access to the side information. We refer to this second class of coding strategies to as side information unaware (SIU) since the correlation between the observed signal and the side information does not need to be known at the encoder. This approach has already been considered in the scalar case in [5], and referred to as "estimate-and-quantize." We consider here the extension to stationary jointly Gaussian random vector processes that will be needed to compute the numerical results presented in Section IV. In the sequel, we denote by $\Phi_{X \mid Y}$ the PSD of the innovation process $X-\mathrm{E}[X \mid Y]$, where $\mathrm{E}[\cdot \cdot \cdot]$ is the conditional expectation.

\section{A. Side Information Aware Coding}

We consider the scenario depicted in Fig. 3(a), where an encoder observes the signal $\mathbf{X}_{2}$ corresponding to the remote source $S_{1,1}$ and outputs a bit stream that appears at a rate of $R_{1}$ bits per second. Based on the received data and the side information $\mathbf{X}_{1}$, the decoder computes a reconstruction $\hat{S}_{1,1}$ inducing the weighted MSE [31, Sec. 4.5.4]

$$
D_{1}=\frac{1}{2 \pi} \int_{-\infty}^{\infty}|A|^{2} \Phi_{E_{1}} d \Omega
$$

where $A$ is the transfer function of a linear and time-invariant operator $\mathcal{A}$ and $E_{1}$ is the reconstruction error process defined as

$$
E_{1}=S_{1,1}-\hat{S}_{1,1} \text {. }
$$

We seek to express the rate-distortion function $D_{1}\left(R_{1}\right)$ which characterizes the optimal tradeoff between the available rate $R_{1}$ 


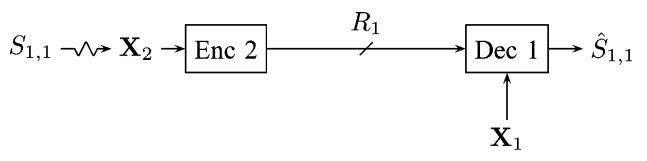

(a)

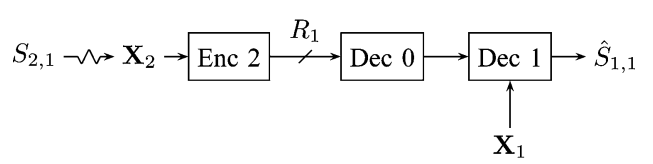

(b)

Fig. 3. Block diagram of the two considered coding strategies. (a) SIA coding. (b) SIU coding.

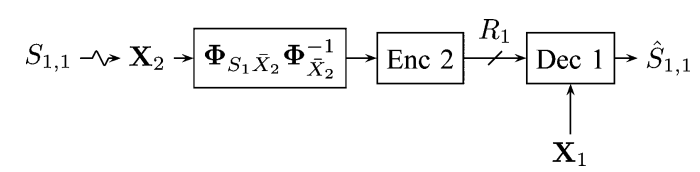

Fig. 4. Optimal encoding architecture with SIA coding. The transmitter breaks into estimation of the remote source $S_{1,1}$ followed by optimal encoding of this estimate. The estimation step corresponds to the Wiener filter designed to estimate $S_{1,1}$ using $\overline{\mathbf{X}}_{2}$.

and the incurred distortion $D_{1}$. The rate-distortion function is given by the following proposition.

Proposition 3.1 (Side Information Aware Coding): The optimal rate-distortion tradeoff with SIA coding is given in parametric form by

$$
\begin{aligned}
R_{1}(\theta)= & \frac{1}{4 \pi} \int_{-\infty}^{\infty} \max \left\{0, \log _{2} \frac{|A|^{2} \Phi_{P_{1}}}{\theta}\right\} d \Omega \\
D_{1}(\theta)= & \frac{1}{2 \pi} \int_{-\infty}^{\infty}|A|^{2} \Phi_{S_{11} \mid X_{1}, X_{2}} d \Omega \\
& +\frac{1}{2 \pi} \int_{-\infty}^{\infty} \min \left\{\theta,|A|^{2} \Phi_{P_{1}}\right\} d \Omega
\end{aligned}
$$

where $\Phi_{P_{1}}=\Phi_{S_{11} \mid X_{1}}-\Phi_{S_{11} \mid X_{1}, X_{2}}$ and $\theta \quad \epsilon$ $\left(0, \operatorname{ess~sup}_{\Omega}|A|^{2} \Phi_{P_{1}}\right]$. Here $R_{1}(\theta)$ is expressed in units of bits per second and $D_{1}(\theta)$ in MSE per second.

Proof: See Appendix A.

Note that the first term in $D_{1}(\theta)$ corresponds to the minimal achievable distortion obtained if both $\mathbf{X}_{1}$ and $\mathbf{X}_{2}$ are available at the decoder to estimate the remote source $S_{1,1}$, i.e., as $R_{1} \rightarrow \infty$. It can be checked that, under our Gaussian assumptions, the availability of the side information at both the encoder and the decoder results in the same rate-distortion tradeoff. This property is inherited from the standard result of Wyner and Ziv [32].

Furthermore, as seen in the proof of Proposition 3.1, an optimal encoding architecture amounts to first estimate the remote source $S_{1,1}$ as if the innovation process $\overline{\mathbf{X}}_{2}=\mathbf{X}_{2}-$ $\mathrm{E}\left[\mathbf{X}_{2} \mid \mathbf{X}_{1}\right]$ were available at the encoder (estimation stage), that is, by passing $\mathbf{X}_{2}$ through the Wiener filter with transfer function $\boldsymbol{\Phi}_{S_{11} \bar{X}_{2}} \Phi_{\bar{X}_{2}}^{-1}$. This estimate is then encoded optimally taking into account the presence of the side information at the decoder (coding stage). This is depicted in Fig. 4.

\section{B. Side Information Unaware Coding}

SIA coding strategies require statistical quantities between signals recorded at the two hearing aids. In a practical setting, this information may be difficult to compute at the encoder as the side information is not observable. We thus consider a transmitter that encodes its observation $\mathbf{X}_{2}$ for a decoder that does not have access to the side information $\mathbf{X}_{1}$. The decoded signal is then provided to the decoder with side information for the reconstruction of the source $S_{1,1}$. Furthermore, the encoder assumes that the process to be estimated at the other end is $S_{2,1}$ and optimizes its coding strategy accordingly. This is illustrated in Fig. 3(b). In this scenario, the encoding strategy reduces to that of a remote source coding problem with a weighted MSE criterion [31, Sec. 4.5.4]. The optimal rate-distortion tradeoff under these conditions is given by the following proposition.

Proposition 3.2 (Side Information Unaware Coding): The optimal rate-distortion tradeoff with SIU coding is given in parametric form by

$$
\begin{aligned}
R_{1}(\theta) & =\frac{1}{4 \pi} \int_{-\infty}^{\infty} \max \left\{0, \log _{2} \frac{|A|^{2} \Phi_{P_{1}}}{\theta}\right\} d \Omega \\
D_{1}(\theta) & \\
= & \frac{1}{2 \pi} \int_{-\infty}^{\infty}|A|^{2}\left(\Phi_{S_{11}}-\frac{\left|\boldsymbol{\Phi}_{S_{11} X_{2}} \boldsymbol{\Phi}_{X_{2}}^{-1} \boldsymbol{\Phi}_{S_{21} X_{2}}^{*}\right|^{2}}{\left|\boldsymbol{\Phi}_{S_{21} X_{2}} \boldsymbol{\Phi}_{X_{2}}^{-1} \boldsymbol{\Phi}_{S_{21} X_{2}}^{*}\right|^{2}}\right) d \Omega \\
& +\frac{1}{2 \pi} \int_{-\infty}^{\infty} \frac{\mid \Phi_{P_{11}}}{\left.\left|\boldsymbol{\Phi}_{S_{21} X_{2}} \boldsymbol{\Phi}_{X_{2}}^{-1} \boldsymbol{\Phi}_{S_{21} X_{2}}^{*}\right|^{2} \boldsymbol{\Phi}_{S_{21} X_{2}}^{*}\right|^{2}} \min ^{\infty}\left\{\theta,|A|^{2} \Phi_{P_{1}}\right\} d \Omega \\
& -\frac{1}{2 \pi} \int_{-\infty}^{\infty}|A|^{2} \\
& \cdot\left[\boldsymbol{\Phi}_{S_{11} X_{1}}-\boldsymbol{\Phi}_{Q_{1}} \max \left\{0,\left(|A|^{2} \Phi_{P_{1}}-\theta\right)\right\}\right] \\
& \cdot\left[\boldsymbol{\Phi}_{X_{1}}-\boldsymbol{\Phi}_{R_{1}} \max \left\{0,\left(|A|^{2} \Phi_{P_{1}}-\theta\right)\right\}\right]^{-1} \\
& \cdot\left[\boldsymbol{\Phi}_{S_{11} X_{1}}-\boldsymbol{\Phi}_{Q_{1}} \max \left\{0,\left(|A|^{2} \Phi_{P_{1}}-\theta\right)\right\}\right]^{*} d \Omega
\end{aligned}
$$

where $\Phi_{P_{1}}=\Phi_{S_{21}}-\Phi_{S_{21} \mid X_{2}}$

$$
\boldsymbol{\Phi}_{Q_{1}}=\frac{\boldsymbol{\Phi}_{S_{11} X_{2}} \boldsymbol{\Phi}_{X_{2}}^{-1} \boldsymbol{\Phi}_{S_{21} X_{2}}^{*} \boldsymbol{\Phi}_{S_{21} X_{2}} \boldsymbol{\Phi}_{X_{2}}^{-1} \boldsymbol{\Phi}_{X_{1} X_{2}}^{*}}{|A|^{2} \Phi_{P_{1}}^{2}}
$$

and

$$
\boldsymbol{\Phi}_{R_{1}}=\frac{\boldsymbol{\Phi}_{X_{1} X_{2}} \boldsymbol{\Phi}_{X_{2}}^{-1} \Phi_{S_{21} X_{2}}^{*} \boldsymbol{\Phi}_{S_{21} X_{2}} \boldsymbol{\Phi}_{X_{2}}^{-1} \Phi_{X_{1} X_{2}}^{*}}{|A|^{2} \Phi_{P_{1}}^{2}}
$$

with $\theta \in\left(0, \operatorname{ess} \sup _{\Omega}|A|^{2} \Phi_{P_{1}}\right]$. Here $R_{1}(\theta)$ is expressed in units of bits per second and $D_{1}(\theta)$ in MSE per second.

Proof: See Appendix B.

In the above equations, the superscript $*$ denotes the conjugate (Hermitian) transpose. It is important to emphasize that the function $D_{1}\left(R_{1}\right)$ described in parametric form by Proposition 3.2 is not the result of a variational problem as stated, for example, in [31, Sec. 2.3]. In particular, it is decreasing but is not necessarily convex. Strictly speaking, we cannot refer to it as a rate-distortion function, but more as the optimal rate-distortion tradeoff for the particular class of coding strategies considered 
here. However, we adopt for simplicity the same notations in Proposition 3.1 and 3.2.

With the SIU scheme, the encoder first computes a weighted MSE estimate of the remote source $S_{2,1}$ using $\mathbf{X}_{2}$ (estimation stage) and then encodes it (coding stage). The method is suboptimal in the sense that i) the remote source $S_{2,1}$ does not correspond to the desired source at hearing aid 1 , namely $S_{1,1}$, and ii) the redundancy between $\mathbf{X}_{1}$ and $\mathbf{X}_{2}$ is not taken into account at the coding stage. It was shown in [25] that, under the considered recording model (1), the loss incurred at the estimation stage is zero if $\mathbf{X}_{2}$ is of dimension one (no dimensionality reduction), or if the PSD matrix of the binaural speech component $\mathbf{S}^{T}=\left[\mathbf{S}_{1}^{T}, \mathbf{S}_{2}^{T}\right]$ is of rank one (single speech source) and that the cross-correlation between $\mathbf{N}_{1}$ and $\mathbf{N}_{2}$ is zero (uncorrelated noise). The difference between SIA and SIU coding may hence not always vanish at high rate. To resolve this asymptotic mismatch, we can consider an hybrid scheme which combines the SIA estimation stage and the SIU coding stage. In this case, the design of the estimation stage still requires statistics which are difficult to estimate in practice. Under the assumption of a single speech source, however, it can be obtained using the alternating optimization method described in [25]. The corresponding optimal rate-distortion tradeoff can be derived in a similar fashion to that of Proposition 3.2 and is thus omitted.

\section{Monaural Gain-Rate Tradeoffs}

Let us apply the results obtained in Section III to the considered hearing aid setup. We adopt the perspective of hearing aid 1 and compute the monaural gain-rate function (2) for the two coding strategies developed previously in a very simple scenario. Far from being realistic, the acoustic environment considered in this section allows us to derive analytical closedform formulas which have many of the features of the gain-rate functions computed numerically in a realistic environment (see Section VI). It hence provides useful insights about the tradeoffs inherent to the considered hearing aid setup.

Let us consider the simplified scenario which solely consists of a speech source surrounded by ambient noise. We assume omnidirectional microphones, neglect the head-shadow effect and work under the far-field assumption. The distortion criterion is the MSE, that is, we set $A \equiv 1$ in (4). Furthermore, the speech and noise sources have flat PSDs over the frequency band $\left[-\Omega_{0}, \Omega_{0}\right]$ such that the $M_{1} \times M_{1}$-dimensional speech and noise PSD matrices of the signals recorded at hearing aid 1 can be expressed as

$$
\boldsymbol{\Phi}_{S_{1}}=P_{S} \mathbf{h}_{1} \mathbf{h}_{1}^{*} \quad \text { and } \quad \boldsymbol{\Phi}_{N_{1}}=P_{N_{1}} \mathbf{I}_{M_{1}}
$$

where $\mathbf{I}_{M_{1}}$ is the $M_{1} \times M_{1}$-dimensional identity matrix. Here $P_{S}=\sigma_{S}^{2} 1_{\left[-\Omega_{0}, \Omega_{0}\right]}$ and $P_{N_{1}}=\sigma_{N_{1}}^{2} 1_{\left[-\Omega_{0}, \Omega_{0}\right]}$ are respectively the power of the speech and the power of the noise at the microphones, $1_{\left[-\Omega_{0}, \Omega_{0}\right]}$ denoting the indicator function on the interval $\left[-\Omega_{0}, \Omega_{0}\right]$. The SNR is thus given by $\gamma_{1}=\sigma_{S}^{2} / \sigma_{N_{1}}^{2}$. The $M_{1}$-dimensional vector

$$
\mathbf{h}_{1}=\left[e^{-j \Omega \tau_{1}}, e^{-j \Omega \tau_{2}}, \ldots, e^{-j \Omega \tau_{M_{1}}}\right]^{T}
$$

contains the acoustic transfer functions from the speech source to the microphones, with $\tau_{m}$ the corresponding propagation delays $\left(m=1,2, \ldots, M_{1}\right)$. With these definitions, $\Phi_{S_{11} \mid X_{1}}$ can be computed as

$$
\begin{aligned}
\Phi_{S_{11} \mid X_{1}} & =\Phi_{S_{11}}-\boldsymbol{\Phi}_{S_{11} X_{1}} \boldsymbol{\Phi}_{X_{1}}^{-1} \boldsymbol{\Phi}_{S_{11} X_{1}}^{*} \\
& =P_{S}-P_{S}^{2} \mathbf{h}_{1}^{*}\left[P_{S} \mathbf{h}_{1} \mathbf{h}_{1}^{*}+P_{N_{1}} \mathbf{I}_{M_{1}}\right]^{-1} \mathbf{h}_{1} \\
& =\sigma_{S}^{2}\left[1+\gamma_{1} \mathbf{h}_{1}^{*} \mathbf{h}_{1}\right]^{-1} 1_{\left[-\Omega_{0}, \Omega_{0}\right]} \\
& =\sigma_{S}^{2}\left[1+M_{1} \gamma_{1}\right]^{-1} 1_{\left[-\Omega_{0}, \Omega_{0}\right]}
\end{aligned}
$$

where the third equality follows from the matrix inversion lemma [33, Sec. 0.7.4], and the fourth one from the fact that $\mathbf{h}_{1}^{*} \mathbf{h}_{1}=M_{1}$. Similarly, $\Phi_{S_{11} \mid X_{1}, X_{2}}$ can be computed as

$$
\Phi_{S_{11} \mid X_{1}, X_{2}}=\sigma_{S}^{2}\left[1+M_{1} \gamma_{1}+M_{2} \gamma_{2}\right]^{-1} 1_{\left[-\Omega_{0}, \Omega_{0}\right]}
$$

where $\gamma_{2}$ denotes the SNR at the microphones of hearing aid 2. Using the PSDs (5) and (6) in Proposition 3.1 yields

$$
\begin{aligned}
& D_{1}^{(a)}\left(R_{1}\right) \\
& =\frac{\Omega_{0}}{\pi} \frac{\sigma_{S}^{2} M_{1} M_{2} \gamma_{1} \gamma_{2}}{1+M_{1} \gamma_{1}+M_{2} \gamma_{2}}\left(1+\frac{M_{2} \gamma_{2}}{1+M_{1} \gamma_{1}} 2^{-2 \pi R_{1} / \Omega_{0}}\right)
\end{aligned}
$$

for $R_{1} \geq 0$. The monaural gain-rate function with SIA coding follows by evaluating (2) using the rate-distortion function (7). We find

$$
\begin{aligned}
& G_{1}^{(a)}\left(R_{1}\right) \\
& =\frac{1+M_{1} \gamma_{1}+M_{2} \gamma_{2}}{1+M_{1} \gamma_{1}}\left(1+\frac{M_{2} \gamma_{2}}{1+M_{1} \gamma_{1}} 2^{-2 \pi R_{1} / \Omega_{0}}\right)^{-1}
\end{aligned}
$$

The gain-rate function obtained with the SIU coding scheme can be obtained similarly. Using Proposition 3.2, we find

$$
\begin{aligned}
& D_{1}^{(u)}\left(R_{1}\right) \\
& =\frac{\Omega_{0} \sigma_{S}^{2}}{\pi M_{1} \gamma_{1}}\left(1+\frac{1+M_{2} \gamma_{2}}{M_{1} \gamma_{1}}\left(1+M_{2} \gamma_{2} 2^{-2 \pi R_{1} / \Omega_{0}}\right)^{-1}\right)^{-1}
\end{aligned}
$$

for $R_{1} \geq 0$. The monaural gain-rate function with SIU coding follows by evaluating (2) using the optimal rate-distortion tradeoff (9). We obtain

$$
\begin{aligned}
& G_{1}^{(u)}\left(R_{1}\right) \\
& =\frac{M_{1} \gamma_{1}}{1+M_{1} \gamma_{1}}+\frac{1+M_{2} \gamma_{2}}{1+M_{1} \gamma_{1}}\left(1+M_{2} \gamma_{2} 2^{-2 \pi R_{1} / \Omega_{0}}\right)^{-1} .
\end{aligned}
$$

To get some insights about the gain provided by the wireless link as a function of the communication bit rate, let us consider the case where the hearing aids are equipped with $M_{1}=M_{2}=$ $M=2$ microphones of equal SNR $\gamma_{1}=\gamma_{2}=\gamma$. We compare, in Fig. 6, the gain achieved by the two coding schemes for different values of $\gamma$. At $20 \mathrm{~dB}$ [Fig. 6(a)], we observe that the SIU coding strategy may lead to a significant loss in terms of noise reduction capability in comparison to the SIA scheme. 


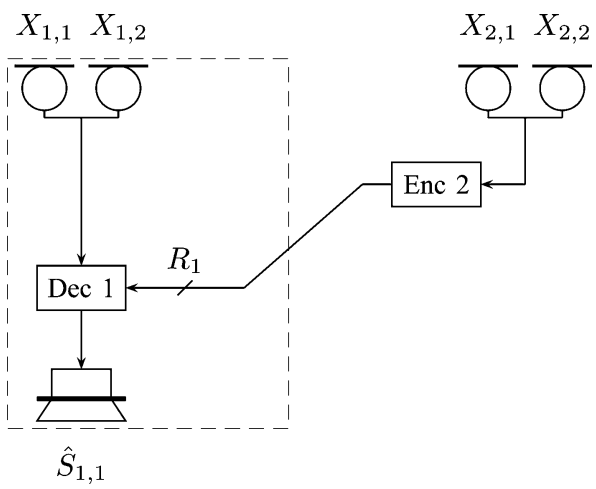

Fig. 5. Monaural hearing aid setup with $M_{1}=M_{2}=2$ microphones from the perspective of hearing aid 1 .

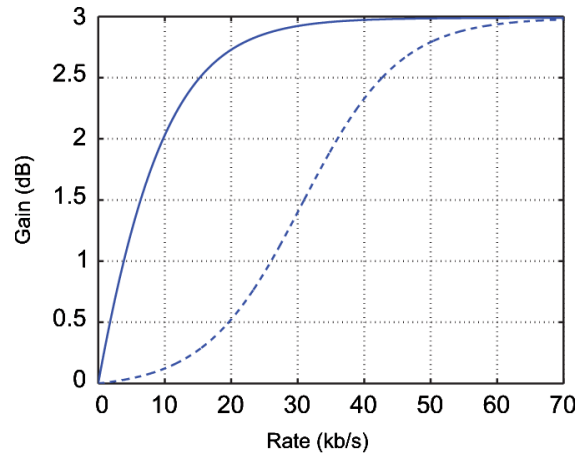

(a)

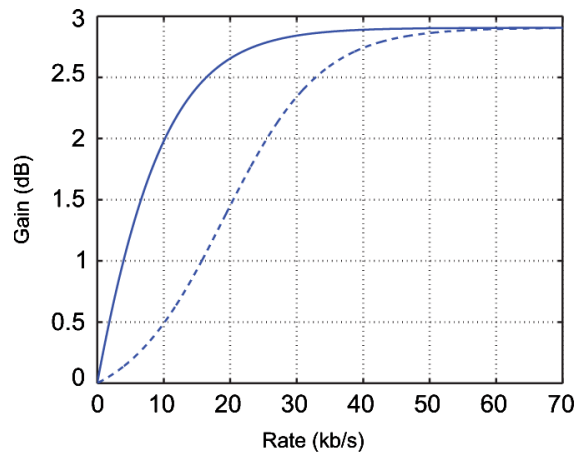

(b)

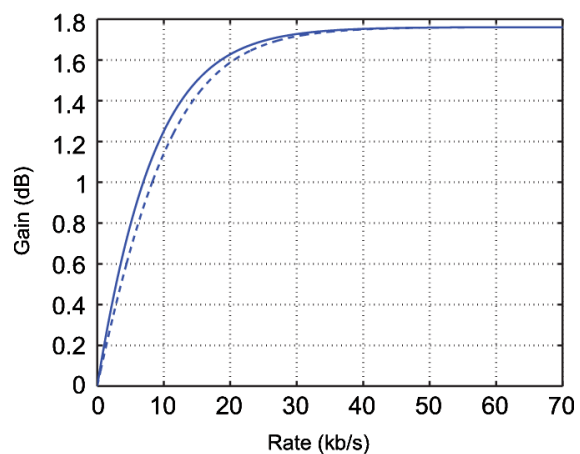

(c)

Fig. 6. Monaural gain-rate functions with SIA coding (solid) and SIU coding (dashed) for different input SNRs $\gamma$. (a) $\gamma=20 \mathrm{~dB}$. (b) $\gamma=10 \mathrm{~dB}$. (c) $\gamma=$ $0 \mathrm{~dB}$. We observe that the gain achieved by taking into account the side information may be significant at high SNR but vanishes in very noisy scenarios.

However, as the input SNR decreases [Fig. 6(b) and (c)], the spatial correlation between the recorded signals decreases significantly and the gap between the SIA and SIU curves vanishes

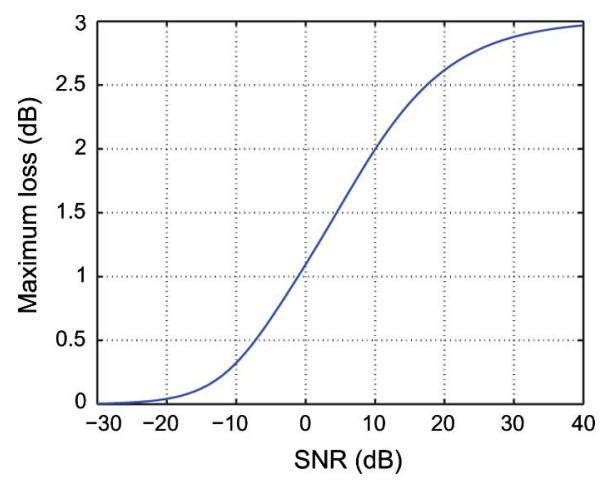

Fig. 7. Maximum loss incurred by the SIU coding strategy over the SIA scheme as a function of the input SNR. We observe that in a highly noisy environment, the gain provided by the SIA approach is rather negligible.

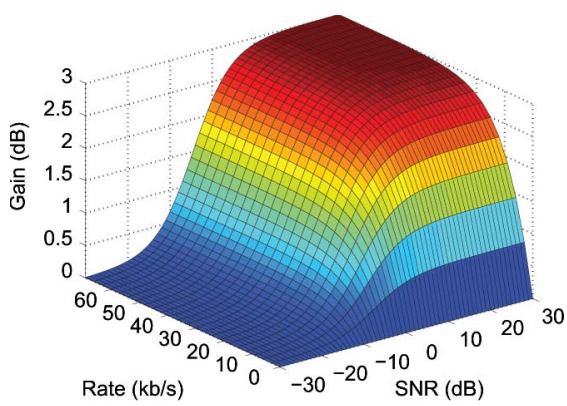

(a)

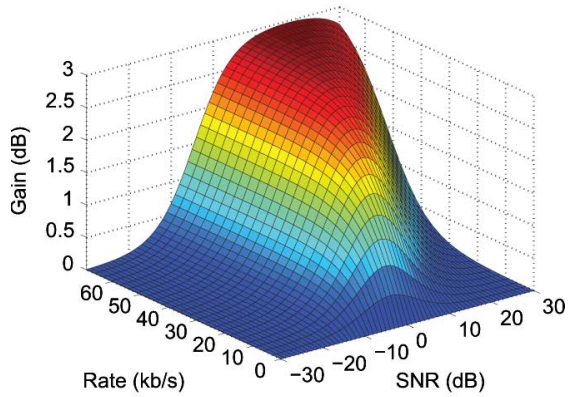

(b)

Fig. 8. Gain provided by the wireless communication link as a function of the communication bit rate and the input SNR with (a) SIA coding and (b) SIU coding. We observe that the gain-rate function in (a) is strictly increasing in both the rate and the SNR while, in case (b), there exists a finite input SNR which provides maximum gain.

for all rates. Using the gain-rate functions (8) and (10), a simple optimization reveals that the maximum loss evaluates as

$$
L_{1}^{\star}=\max _{R_{1}} \frac{G_{1}^{(a)}\left(R_{1}\right)}{G_{1}^{(u)}\left(R_{1}\right)}=\frac{(2 M \gamma+1)(M \gamma+1)}{(M \gamma+\sqrt{M \gamma+1})^{2}},
$$

and is attained at

$$
R_{1}^{\star}=\arg \max _{R_{1}} \frac{G_{1}^{(a)}\left(R_{1}\right)}{G_{1}^{(u)}\left(R_{1}\right)}=\frac{\Omega_{0}}{2 \pi} \log _{2}(1+\sqrt{M \gamma+1}) .
$$

The maximum loss (11) is plotted as a function of the input SNR $\gamma$ in Fig. 7. The result suggests that the use of SIA coding strategies is uninteresting in very noisy scenarios.

In Fig. 8, we plot the noise reduction gain provided by the wireless link as a function of the communication bit rate $R_{1}$ 
and the input SNR $\gamma$. The following remarks are at hand. As $R_{1} \rightarrow \infty$, both $G_{1}^{(a)}\left(R_{1}\right)$ and $G_{1}^{(u)}\left(R_{1}\right)$ remain bounded and correspond to the gain obtained when there is no rate constraint. At high SNR, this gain approaches $10 \log _{10}(L) \mathrm{dB}$, where $L=$ 2 relates to the fact that the wireless link allows to double the number of available microphones. We also notice that, in this scenario, the results depend neither on the actual position of the source nor on the geometrical properties of the hearing aid setup. This results from the far-field assumption and the fact that the noise is uncorrelated across microphones. While the function $G_{1}^{(a)}$ is strictly increasing in both $R_{1}$ and $\gamma$, the behavior of $G_{1}^{(u)}$ is slightly different. It is strictly increasing in $R_{1}$ but, for any communication bit rate, there exists a finite $\operatorname{SNR} \gamma^{\star}$, easily evaluated from (10) as $\gamma^{\star}=2^{\pi R_{1} / \Omega_{0}}$, which provides maximum gain [see Fig. 8(b)]. This fact may be explained by the following observation. As the SNR increases, the availability of signals recorded from both hearing aids permits a better estimation of the desired source. However, the observed signals also become more correlated. In the scenario where the side information is neglected at the encoder, the communication resources are thus used to transmit redundant information, hence preventing significant noise reduction. The observed "optimum" follows from these two opposite behaviors. Note also that, under the present assumptions (single speech source and uncorrelated noise), the estimation stage of the SIU scheme does not incur any loss compared to that of the SIA scheme. The apparent differences are solely due to the suboptimality of the coding stage. In this scenario, the hybrid architecture discussed in Section III is thus equivalent to the SIU strategy.

\section{Binaural Gain-Rate TradeofFS}

Let us now turn our attention to the general binaural setup depicted in Fig. 2. A natural question that arises in this context is that of the optimal rate allocation between the two hearing instruments. More precisely, assume that you are given a total bit budget of $R$ bits per second, how should this be allocated to $R_{1}$ and $R_{2}$ to minimize the sum distortion $D=D_{1}+D_{2}$, hence maximizing the binaural gain $G(R)$ given by (3)? To this end, observe that the hearing aid setup depicted in Fig. 2 corresponds to two separate source coding problems. The overall optimal rate-distortion tradeoff $D(R)$ can thus be obtained by means of the minimization task

$$
\begin{array}{ll} 
& D(R)=\min _{R_{1}, R_{2}} D_{1}\left(R_{1}\right)+D_{2}\left(R_{2}\right), \\
\text { s.t. } & R_{1}+R_{2} \leq R \text { and } \quad R_{1}, R_{2} \geq 0 .
\end{array}
$$

We consider the simple scenario studied in Section IV as a means to obtain an analytical characterization of the optimal policy for the rate allocation between the hearing aids. In the sequel, we assume, without lost of generality, that $\gamma_{1} \geq \gamma_{2}$.

Let us first consider the SIA coding scheme. In this case, the minimization problem (13) can be restated as

$$
\begin{array}{ll} 
& D^{(a)}(R)=\min _{R_{1}, R_{2}} f\left(R_{1}, R_{2}\right), \\
\text { s.t. } & g_{k}\left(R_{1}, R_{2}\right) \leq 0 \text { for } k=1,2,3
\end{array}
$$

with $f, g_{k}: \mathbb{R}^{2} \rightarrow \mathbb{R}$ defined by

$$
\begin{aligned}
f\left(R_{1}, R_{2}\right) & =D_{1}^{(a)}\left(R_{1}\right)+D_{2}^{(a)}\left(R_{2}\right), \\
g_{1}\left(R_{1}, R_{2}\right) & =R_{1}+R_{2}-R, \\
g_{2}\left(R_{1}, R_{2}\right) & =-R_{1},
\end{aligned}
$$

and

$$
g_{3}\left(R_{1}, R_{2}\right)=-R_{2}
$$

where $D_{1}^{(a)}\left(R_{1}\right)$ and $D_{2}^{(a)}\left(R_{2}\right)$ are computed from (7). Since the functions $f$ and $g_{k}$ are convex and differentiable on $\mathbb{R}^{2}$, the optimal solution $\left(R_{1}^{\star}, R_{2}^{\star}\right)$ follows from the Karush-Kuhn-Tucker conditions [34, Sec. 5.5.3]. We obtain

$$
R_{1}^{\star}= \begin{cases}\frac{1}{2}(R-\bar{R}) & \text { if } R \geq \bar{R}^{(a)} \\ 0 & \text { otherwise }\end{cases}
$$

and $R_{2}^{\star}=R-R_{1}^{\star}$. The threshold rate $\bar{R}^{(a)}$ is given by

$$
\bar{R}^{(a)}=\bar{R}=\frac{\Omega_{0}}{2 \pi} \log _{2} \frac{M_{1} \gamma_{1}\left(M_{1} \gamma_{1}+1\right)}{M_{2} \gamma_{2}\left(M_{2} \gamma_{2}+1\right)} .
$$

The binaural gain-rate function $G^{(a)}(R)$ then follows from the definition (3) using the optimal rate-distortion tradeoff $D^{(a)}(R)=D_{1}^{(a)}\left(R_{1}^{\star}\right)+D_{2}^{(a)}\left(R_{2}^{\star}\right)$ that can be computed from (7). We find

$$
G^{(a)}(R)=C_{1}\left[2+\frac{2 M_{2} \gamma_{2}}{M_{1} \gamma_{1}+1} 2^{-\pi / \Omega_{0}(R-\bar{R})}\right]^{-1}
$$

if $R \geq \bar{R}^{(a)}$, and

$$
\begin{aligned}
G^{(a)}(R)=C_{1}\left[1+\frac{M_{1} \gamma_{1}+}{M_{1} \gamma_{1}+1} \gamma_{2}+1\right. \\
\left.\quad+\frac{M_{2} \gamma_{2}}{M_{1} \gamma_{1}+1} 2^{-2 \pi / \Omega_{0}(R-\bar{R})}\right]^{-1}
\end{aligned}
$$

otherwise. The constant $C_{1}$ is defined as

$$
C_{1}=1+\frac{M_{2} \gamma_{2}}{M_{1} \gamma_{1}+1}+\frac{M_{1} \gamma_{1}+M_{2} \gamma_{2}+1}{M_{2} \gamma_{2}+1} .
$$

Let us now consider the SIU coding scheme. In this case, the minimization problem (13) cannot be solved using the Karush-Kuhn-Tucker conditions since the involved optimal rate-distortion tradeoffs $D_{1}^{(u)}\left(R_{1}\right)$ and $D_{2}^{(u)}\left(R_{2}\right)$ given by (9) are not necessarily convex. However, we note that $D_{1}^{(u)}\left(R_{1}\right)$ and $D_{2}^{(u)}\left(R_{2}\right)$ are strictly decreasing functions. The inequality $R_{1}^{\star}+R_{2}^{\star} \leq R$ is thus active at the optimum, otherwise we can always increase $R_{1}^{\star}$ or $R_{2}^{\star}$ by $R-R_{1}^{\star}-R_{2}^{\star}$ and lower the resulting distortion. The optimization task (13) thus reduces to

$$
D^{(u)}(R)=\min _{R_{1}} D_{1}^{(u)}\left(R_{1}\right)+D_{2}^{(u)}\left(R-R_{1}\right),
$$

s.t. $0 \leq R_{1} \leq R$. 
A tedious but relatively straightforward functional analysis reveals that the optimal solution $\left(R_{1}^{\star}, R_{2}^{\star}\right)$ is given by

$R_{1}^{\star}$

$$
= \begin{cases}\frac{1}{2}(R-\bar{R})-\frac{\Omega_{0}}{2 \pi} \log _{2} \frac{1-C_{2} 2^{-\pi / \Omega_{0}(R+\bar{R})}}{1-C_{2} 2^{-\pi / \Omega_{0}(R-\bar{R})}} & \text { if } R \geq \bar{R}^{(u)} \\ 0 & \text { otherwise }\end{cases}
$$

and $R_{2}^{\star}=R-R_{1}^{\star}$, where $\bar{R}$ is given by (14) and the constant $C_{2}$ is defined as

$$
C_{2}=\frac{M_{1} M_{2} \gamma_{1} \gamma_{2}}{M_{1} \gamma_{1}+M_{2} \gamma_{2}+1} .
$$

In this case, the threshold rate $\bar{R}^{(u)}$ can be expressed as

$$
\begin{aligned}
\bar{R}^{(u)}=\max \{ & 0, \bar{R}+\frac{\Omega_{0}}{\pi} \log _{2} \frac{C_{2}+1}{2} \\
& \left.\cdot\left(1+\sqrt{1-\frac{4 C_{2}}{\left(C_{2}+1\right)^{2}} 2^{-2 \pi / \Omega_{0} \bar{R}}}\right)\right\} .
\end{aligned}
$$

The binaural gain-rate function $G^{(u)}(R)$ then follows from the definition (3) using the optimal rate-distortion tradeoff $D^{(u)}(R)=D_{1}^{(u)}\left(R_{1}^{\star}\right)+D_{2}^{(u)}\left(R_{2}^{\star}\right)$ that can be computed from (9). We find

$$
\begin{aligned}
G^{(u)}(R)= & C_{3}\left(M_{1} \gamma_{1}+M_{2} \gamma_{2}+2\right)\left[2^{-2 \pi R / \Omega_{0}}-\left(\frac{1}{C_{2}}\right)^{2}\right] \\
\cdot & {\left[\left(M_{1} \gamma_{1}+M_{2} \gamma_{2}\right) 2^{-2 \pi R / \Omega_{0}}\right.} \\
& \left.-2 \sqrt{\frac{1}{C_{3}}} 2^{-\pi R / \Omega_{0}}-\frac{2}{C_{2}}\right]^{-1}
\end{aligned}
$$

if $R \geq \bar{R}^{(u)}$, and

$$
\begin{aligned}
G^{(u)}(R) & \\
= & {\left[1+\frac{M_{1} \gamma_{1}+1}{M_{2} \gamma_{2}+1}\right] } \\
& \cdot\left[1+\frac{\left(1+M_{1} \gamma_{1}\right)\left(1+M_{1} \gamma_{1} 2^{-2 \pi / \Omega_{0} R}\right)}{\left(1+M_{1} \gamma_{1}+M_{2} \gamma_{2}\right)\left(1+C_{2} 2^{-2 \pi / \Omega_{0} R}\right)}\right]^{-1}
\end{aligned}
$$

otherwise. The constant $C_{3}$ is defined as

$$
C_{3}=\frac{M_{1} M_{2} \gamma_{1} \gamma_{2}}{\left(M_{1} \gamma_{1}+1\right)\left(M_{2} \gamma_{2}+1\right)} .
$$

In Fig. 9(a), we plot an example of binaural gain-rate functions for $\gamma_{1}=10 \mathrm{~dB}, \gamma_{2}=0 \mathrm{~dB}$ and $M_{1}=M_{2}=M=2$ microphones. The corresponding rate allocation is depicted in Fig. 9(b). The rate allocation strategies derived above suggest that the hearing device with smaller SNR does not transmit any data unless the total available bit rate is larger than a given threshold. Below this rate, the noisiest device benefits exclusively from the available bandwidth. At equal SNR $\left(\gamma_{1}=\gamma_{2}=\right.$ $\gamma$ ), the threshold rate $\bar{R}^{(a)}$ of the SIA coding scheme is equal to zero. In other words, the communication medium is evenly shared between the two hearing aids for all rates. By contrast, the threshold rate with SIU coding reduces to

$\bar{R}^{(u)}=\max \left\{0, \frac{\Omega_{0}}{\pi} \log _{2} C_{2}\right\}=\max \left\{0, \frac{\Omega_{0}}{\pi} \log _{2} \frac{M^{2} \gamma^{2}}{2 M \gamma+1}\right\}$

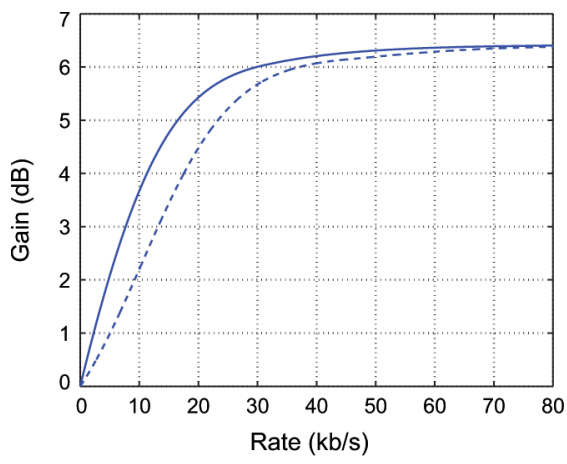

(a)

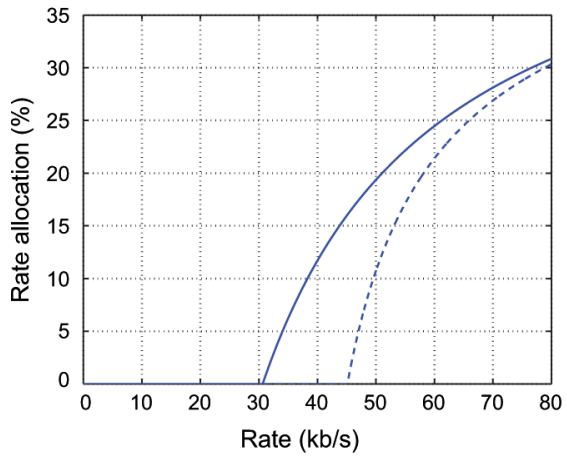

(b)

Fig. 9. Binaural communication using SIA coding (solid) and SIU coding (dashed). (a) Binaural gain-rate functions. (b) Percentage of the total bit rate benefitting to hearing aid 1 . Here $\gamma_{1}=10 \mathrm{~dB}$ and $\gamma_{2}=0 \mathrm{~dB}$. We observe that, for small enough rates, the noisiest device benefits exclusively from the wireless link.

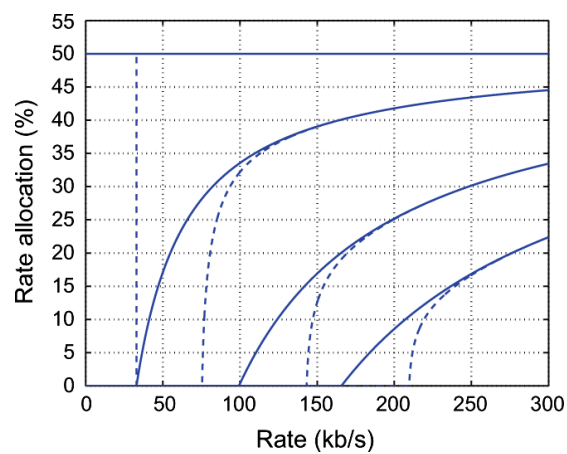

Fig. 10. Percentage of the total bit rate benefiting to hearing aid 1 with SIA coding (solid) and SIU coding (dashed). The different sets of curves correspond to $\left(\gamma_{1}, \gamma_{2}\right) \in\{(10,10),(20,10),(40,10),(60,10)\} \mathrm{dB}$ (left to right). We observe that the threshold rate for SIU coding is larger than that of SIA coding.

such that it is greater than zero for large enough SNR. Surprisingly, in this seemingly symmetric scenario, the communication bandwidth may hence not be equally shared. Fig. 10 depicts the percentage of the total bit rate benefiting to hearing aid 1 for different input SNRs. With SIU coding at equal SNR, we observe a sharp transition between two policies, namely unidirectional communication and equal rate allocation. In the former case, it can be checked that the hearing aid benefitting from the wireless link can be chosen arbitrarily. We also note that the SIU threshold rate is larger than that of the SIA coding scheme. Finally, note that, as in Section IV, the hybrid scheme would not improve upon the SIU scheme under the present assumptions. 


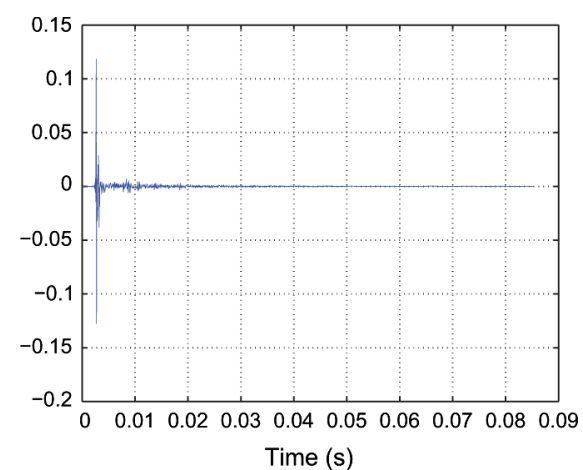

(a)

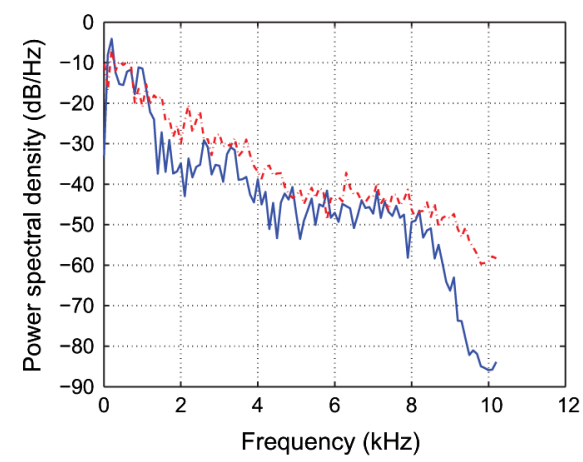

(b)

Fig. 11. Experimental data. (a) Typical head-related impulse response. (b) Example of speech (solid) and noise (dashed-dotted) power spectral densities. The noise source is a multitalker babble noise.

\section{Simulation RESULTS}

We present numerical results obtained from acoustic data recorded in a realistic environment. The recording setup is described in Section VI-A along with the considered distortion measure. In Section VI-B, different noise configurations are simulated as a means to quantify the real benefit of SIA coding over SIU coding in a practical scenario. Section VI-C then explores the gain provided by the increased spatial extent offered by collaborating hearing aids. Finally, Section VI-D discusses optimal rate allocation strategies.

\section{A. Setup}

Two hearing aids, each equipped with $M_{1}=M_{2}=2$ omni-directional microphones at a distance of approximately $1 \mathrm{~cm}$, have been mounted on a dummy head in a room with reverberation time ${ }^{2} R T_{60} \approx 120 \mathrm{~ms}$. The head-related transfer functions (HRTF) for the four microphones have been measured every $15^{\circ}$ in the horizontal plane for a loudspeaker at a distance of $1 \mathrm{~m}$. Here, the angles are measured clockwise and the zero angle corresponds to the front.The sampling frequency is set to $20.48 \mathrm{kHz}$. The acoustic scene is synthesized using the measured HRTFs. The speech component in (1) corresponds to a single speech source at $0^{\circ}$, i.e., it is obtained as the convolution of a speech signal with the corresponding head-related impulse response (HRIR). A typical HRIR is depicted in Fig. 11(a).

\footnotetext{
${ }^{2}$ The reverberation time $R T_{60}$ is defined as the time required for the sound
} level to decrease by $60 \mathrm{~dB}$ after the original sound source is removed.

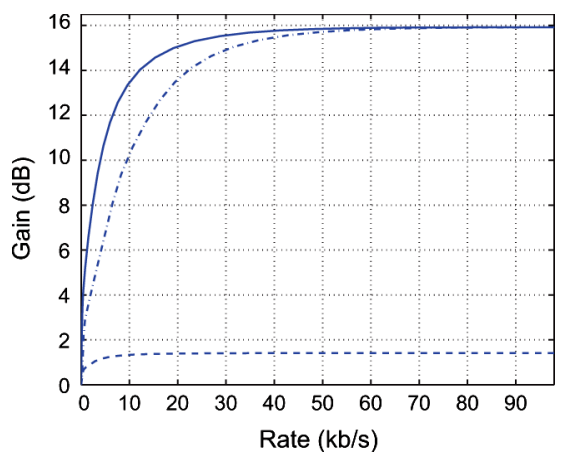

Fig. 12. Monaural gain-rate functions with SIA coding (solid), SIU coding (dashed) and the hybrid scheme (dashed-dotted), in the presence of one interferer at $90^{\circ}$. In this scenario, a significant loss is incurred by the SIU scheme owing to the suboptimality of the estimation stage.

The noise component consists of a stationary white Gaussian ambient noise along with one or more interfering point sources of equal power at different azimuths. The PSD of the speech component is estimated using $3 \mathrm{~s}$ of a sentence of the HINT database [35]. The PSD of the interferers are each computed using a 3-s segment of multitalker babble noise available in the NOISEX-92 database [36]. The HINT database is commonly used for speech intelligibility studies and the NOISEX-92 database contains excerpts from various realistic noisy environments. An example of speech and noise PSDs is plotted in Fig. 11(b). The power of the involved sources is adjusted such as to meet a desired SNR and interference-to-noise ratio (INR) of $20 \mathrm{~dB}$ at the front microphone of hearing aid 1, i.e., the signal-to-interference ratio is equal to $0 \mathrm{~dB}$.

To assess the noise reduction improvement provided by collaborating hearing aids, the transfer function $A$ used in the weighted mean-squared error (4) expresses the relative importance of the frequency $\Omega$ for speech intelligibility, as defined in [37]. Note also that, unless otherwise stated, the results presented in this section are computed from the perspective of hearing aid 1.

\section{B. SIA versus SIU Coding}

Fig. 12 depicts the monaural gain-rate functions for a single interfering source at $90^{\circ}$. In this scenario, the estimation stage of the SIU encoder is strictly suboptimal compared to that of the SIA encoder (more than one microphone and correlated noise). The loss incurred by the SIU scheme is thus significant, in particular in the high-rate regime. The hybrid scheme resolves this asymptotic mismatch but still incurs a penalty over the SIA scheme owing to the suboptimality of its coding stage. As the number of interfering sources increases, however, the correlation between the signals recorded at the hearing aids decreases. Fig. 13 plots the maximum loss (11) over all rates for different noise configurations. We observe that the performance gap between the two coding strategies is reduced significantly, corroborating the analytical results obtained for the simple acoustic model analyzed in Section IV. While SIA coding strategies may provide large gains in simple acoustic environments, their use is rather questionable in more noisy scenarios, for example, a discussion in a crowded room. 


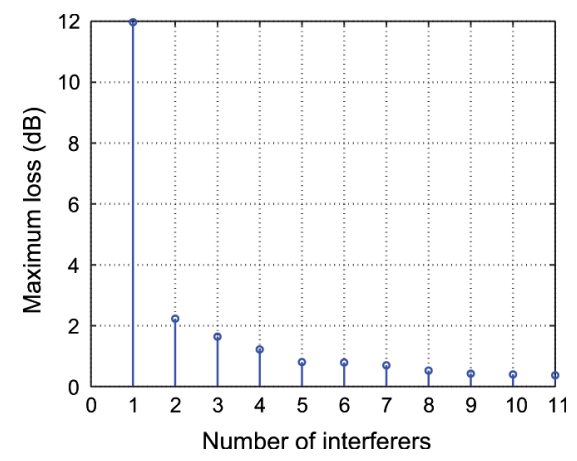

Fig. 13. Maximum loss incurred by the SIU coding strategy over the SIA scheme as a function of the number of interfering point sources. The first interferer is positioned at $30^{\circ}$ and the subsequent ones every $30^{\circ}$ clockwise. The SNR and INR are kept fixed for all configurations. We observe that the loss associated with the use of the SIU scheme is negligible in a very noisy environment.

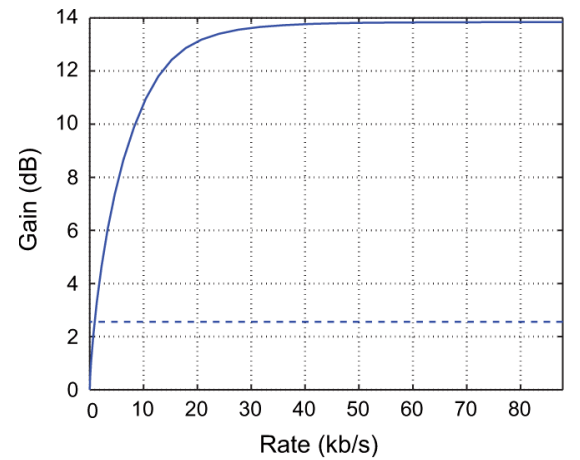

(a)

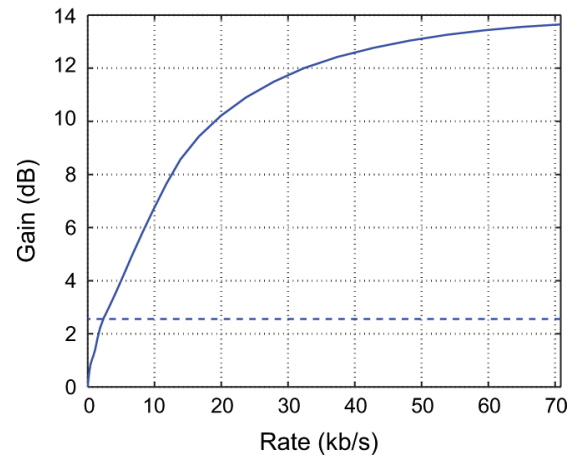

(b)

Fig. 14. Comparison of the gains obtained for a monaural (dashed) and binaural (solid) microphone configuration with one interferer at 15 . The coding scheme is (a) SIA and (b) SIU. In the monaural case, we use two microphones of the same hearing device; there is no rate constraint. In the binaural case, we use the first microphone of each hearing aid. We observe that the use of binaural signals provide large beamforming gains.

\section{Spatial Extent}

The use of a wireless link allows to combine signals from microphones that together form an array with greater spatial extent, enabling better beamforming resolution. To illustrate this fact, Fig. 14 depicts the monaural gain-rate functions obtained using the first microphone of each hearing device (binaural configuration), typically $20 \mathrm{~cm}$ apart. We compare these gains with that achieved using the two microphones directly embedded in hearing aid 1 (monaural configuration). We observe that, for large enough communication rates, significant gains can be achieved by exploiting the inter-aural distance.

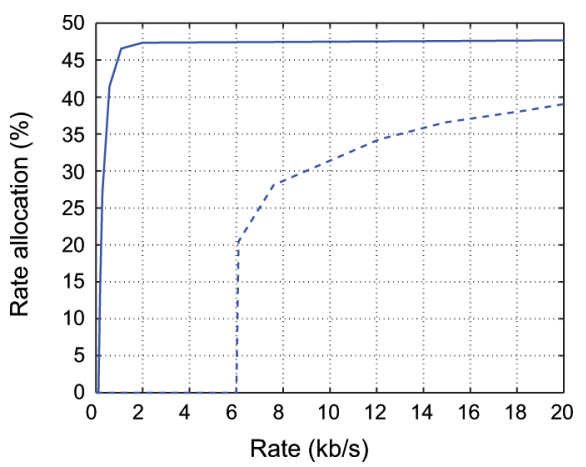

(a)

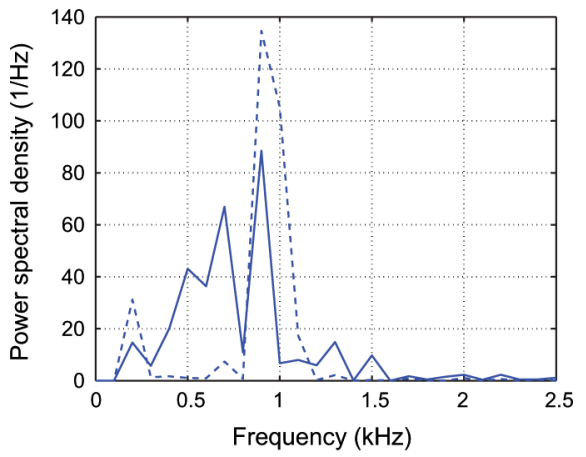

(b)

Fig. 15. Rate allocation with one interferer at 90 using SIA coding (solid) and SIU coding (dashed). (a) Percentage of the total bit rate benefiting to hearing aid 1. Hearing aid 1 is subject to less disturbances and thus benefits of less communication resources. (b) Power spectral densities used for the reverse "water-filling" allocation strategy.

At very low bit rates, however, the quality of the transmitted signal is not sufficient for the binaural configuration to compete with the monaural one, since the latter does not undergo any communication rate constraints. Note that, more generally, the beamforming capability of the system can be evaluated by means of its directivity pattern; the system is optimized for a specific acoustic scenario and the response to a monochromatic source is computed for all possible propagating directions. With our rate constraint, however, the computation of such a directivity pattern would require to derive the distortion achieved when the input statistics differ from those used to design the associated encoding and decoding algorithms. Hence, the optimal rate-distortion tradeoffs derived in Section III cannot be used for this purpose.

\section{Rate Allocation}

As mentioned previously, the problem of collaborating hearing aids raises two important questions related to the allocation of the available communication resources. In a binaural setup, how should the total bit rate be shared between the two hearing devices? How should then each hearing aid allocate its own resources across the signal bandwidth?

The first issue was addressed in Section V in a simple, yet insightful, scenario. The intuition is that the hearing device that undergoes the strongest disturbances benefit from the total available bit rate up to a certain threshold, above which the communication resources start being shared. Fig. 15 depicts the rate allocation in the case of an interfering point source at $90^{\circ}$. Owing 
to the head-shadow effect, hearing aid 2 experiences more noise than hearing aid 1 . This fact is even more apparent in acoustic environment with low reverberation, such as the one considered here. As it can be observed, the percentage of the total available bit rate benefiting to hearing aid 1 remains null up to the threshold rate $\bar{R}^{(a)} \approx 0.1 \mathrm{~kb} / \mathrm{s}$ with SIA coding, and $\bar{R}^{(u)} \approx$ $6 \mathrm{~kb} / \mathrm{s}$ with SIU coding. The rate allocation strategy is thus similar to that of the scenario analyzed in Section V for which the optimal rule has been derived.

The second issue pertains to the allocation of the bit rate across the frequency support of the transmitted signal, at one hearing device. Here, the optimal strategy directly follows from Propositions 3.1 and 3.2 which suggest to allocate the available bit rate according to the well-known reverse "water-filling" principle [38, Sec. 13.3.3], i.e., such that the frequency bands with higher energy are allocated more bit rate. For illustration purpose, we plot in Fig. 15(b) the one-sided PSDs used at hearing aid 1 for rate allocation with SIA and SIU coding in the presence of a single interferer at $45^{\circ}$. In this example, the frequency band with center frequency $700 \mathrm{~Hz}$ is significant for the rate allocation with SIA coding, while it has little importance in the SIU scheme.

\section{CONCLUSION}

We have investigated the noise reduction provided by hearing aids allowed to collaborate using a rate-constrained wireless link in order to form a binaural system. The problem has been identified and solved from an information-theoretic standpoint. To this end, the optimal rate-distortion tradeoffs corresponding to two coding strategies have been derived. Under simplified assumptions on the acoustic environment, we have precisely assessed the noise reduction capability of the system as a function of the available communication bit rate, in both a monaural and a binaural configuration. In particular, it was shown that the loss incurred by neglecting the binaural correlation for coding purpose is rather negligible in a very noisy scenario. Optimal rate allocation strategies between the hearing aids have also been computed. Finally, simulation results have confirmed the potential of our approach in a realistic scenario.

\section{APPENDIX A}

\section{PROOF OF PROPOSITION 3.1}

The rate-distortion function has been derived in [4, Th. 2]. Our goal is to evaluate it under our jointly Gaussian assumption. To this end, we proceed similarly to [39, Th. 2]. We first establish that no better rate-distortion tradeoff can be hoped for (converse part) and then provide an encoding architecture that achieves it (achievability part).

Converse: The rate-distortion function follows as [4, Th. 2]

$$
R_{1}=\min _{U \in \mathcal{U}} I\left(\mathbf{X}_{2} ; U \mid \mathbf{X}_{1}\right)
$$

where $\mathcal{U}$ denotes the set of random processes such that $\left(S_{1,1}, \mathbf{X}_{1}\right) \longleftrightarrow \mathbf{X}_{2} \longleftrightarrow U$ and

$$
\mathrm{E}\left[\left\|\mathcal{A}\left(S_{1,1}-\mathrm{E}\left[S_{1,1} \mid \mathbf{X}_{1}, U\right]\right)\right\|^{2}\right] \leq D_{1} .
$$

In the above notation, $I(\cdot, \cdot)$ is the mutual information and $X \longleftrightarrow Y \longleftrightarrow Z$ denotes a Markov chain. It can be easily checked that a lower bound to the above minimization task is obtained as

$$
R_{1}=\min _{p\left(u \mid \mathbf{x}_{1}, \mathbf{x}_{2}\right)} I\left(\mathbf{X}_{2} ; U \mid \mathbf{X}_{1}\right)
$$

and $\mathrm{E}\left[\left\|\mathcal{A}\left(S_{1,1}-U\right)\right\|^{2}\right] \leq D_{1}$, where now the minimization is over all conditional distribution $p\left(u \mid \mathbf{x}_{1}, \mathbf{x}_{2}\right)$. We have that

$$
I\left(\mathbf{X}_{2} ; U \mid \mathbf{X}_{1}\right)=I\left(\overline{\mathbf{X}}_{2} ; \bar{U} \mid \mathbf{X}_{1}\right) \geq I\left(\mathrm{E}\left[S_{1,1} \mid \overline{\mathbf{X}}_{2}\right] ; \bar{U} \mid \mathbf{X}_{1}\right)
$$

where $\overline{\mathbf{X}}_{2}=\mathbf{X}_{2}-\mathrm{E}\left[\mathbf{X}_{2} \mid \mathbf{X}_{1}\right]$ and $\bar{U}=U-\mathrm{E}\left[S_{1,1} \mid \mathbf{X}_{1}\right]$. Moreover, the distortion can be split as

$$
\begin{aligned}
\mathrm{E}\left[\left\|\mathcal{A}\left(S_{1,1}-U\right)\right\|^{2}\right] \stackrel{(a)}{=} & \mathrm{E}\left[\left\|\mathcal{A}\left(S_{1,1}-\mathrm{E}\left[S_{1,1} \mid \mathbf{X}_{1}, \mathbf{X}_{2}\right]\right)\right\|^{2}\right] \\
& +\mathrm{E}\left[\left\|\mathcal{A}\left(\mathrm{E}\left[S_{1,1} \mid \mathbf{X}_{1}, \mathbf{X}_{2}\right]-U\right)\right\|^{2}\right] \\
\stackrel{(b)}{=} & \mathrm{E}\left[\left\|\mathcal{A}\left(S_{1,1}-\mathrm{E}\left[S_{1,1} \mid \mathbf{X}_{1}, \mathbf{X}_{2}\right]\right)\right\|^{2}\right] \\
& +\mathrm{E}\left[\left\|\mathcal{A}\left(\mathrm{E}\left[S_{1,1} \mid \overline{\mathbf{X}}_{2}\right]-\bar{U}\right)\right\|^{2}\right]
\end{aligned}
$$

where (a) follows from the orthogonality principle and the fact that $U$ is a function of $\mathbf{X}_{1}$ and $\mathbf{X}_{2}$, and (b) from the equality $\mathrm{E}\left[S_{1,1} \mid \mathbf{X}_{1}, \mathbf{X}_{2}\right]=\mathrm{E}\left[S_{1,1} \mid \mathbf{X}_{1}\right]+\mathrm{E}\left[S_{1,1} \mid \overline{\mathbf{X}}_{2}\right]$ in the jointly Gaussian case. A lower bound is thus obtained as

$$
R_{1}=\min _{p\left(\bar{u} \mid \mathbf{x}_{1}, \mathbf{x}_{2}\right)} I\left(\mathrm{E}\left[S_{1,1} \mid \overline{\mathbf{X}}_{2}\right] ; \bar{U} \mid \mathbf{X}_{1}\right)
$$

such that

$$
\begin{aligned}
\mathrm{E}\left[\left\|\mathcal{A}\left(\mathrm{E}\left[S_{1,1} \mid \overline{\mathbf{X}}_{2}\right]-\bar{U}\right)\right\|^{2}\right] \\
\\
\leq D_{1}-\mathrm{E}\left[\left\|\mathcal{A}\left(S_{1,1}-\mathrm{E}\left[S_{1,1} \mid \mathbf{X}_{1}, \mathbf{X}_{2}\right]\right)\right\|^{2}\right] .
\end{aligned}
$$

Since $\mathrm{E}\left[S_{1,1} \mid \overline{\mathbf{X}}_{2}\right]$ is independent of $\mathbf{X}_{1}$, the optimal $\bar{U}$ does not depend on the particular value of $\mathbf{X}_{1}$. The conditioning in (16) can thus be omitted and the problem reduces to a direct encoding with minimum weighted mean-squared error [40] of the source $P_{1}=\mathrm{E}\left[S_{1,1} \mid \overline{\mathbf{X}}_{2}\right]$ whose PSD can be computed as

$$
\Phi_{P_{1}}=\Phi_{S_{11}}-\Phi_{S_{11} \mid \bar{X}_{2}}=\Phi_{S_{11} \mid X_{1}}-\Phi_{S_{11} \mid X_{1}, X_{2}} .
$$

The lower bound thus directly follows from the corresponding rate-distortion function and yields the claimed formula.

Achievability: Let us consider the encoding architecture depicted in Fig. 4. We first apply to the process $\mathbf{X}_{2}$ the linear operator $\mathcal{W}$ with transfer function $\boldsymbol{\Phi}_{S_{11} \bar{X}_{2}} \boldsymbol{\Phi}_{\bar{X}_{2}}^{-1}$. The obtained signal $\mathcal{W} \mathbf{X}_{2}$ is then optimally encoded provided that $\mathbf{X}_{1}$ is available at the decoder. The corresponding rate-distortion function can be directly derived from [41] using the Toeplitz distribution theorem [42, Ch. 7] and a weighted MSE criterion. It actually corresponds to the rate-distortion function of the innovation process $\mathcal{W} \mathbf{X}_{2}-\mathrm{E}\left[\mathcal{W} \mathbf{X}_{2} \mid \mathbf{X}_{1}\right]=\mathcal{W} \overline{\mathbf{X}}_{2}$ whose PSD is given by (17). The rate achieved by this scheme hence follows from [40] and corresponds to $R_{1}(\theta)$. Let us denote by $Y_{2}$ the process received at the decoder. By the orthogonality principle, the distortion achieved by this method can be decomposed as

$$
\begin{aligned}
\mathrm{E}\left[\left\|\mathcal{A}\left(S_{1,1}-\mathrm{E}\left[S_{1,1} \mid \mathbf{X}_{1}, Y_{2}\right]\right)\right\|^{2}\right] \\
=\mathrm{E}\left[\left\|\mathcal{A}\left(S_{1,1}-\mathrm{E}\left[S_{1,1} \mid \mathbf{X}_{1}, \mathbf{X}_{2}\right]\right)\right\|^{2}\right] \\
\quad+\mathrm{E}\left[\left\|\mathcal{A}\left(\mathrm{E}\left[S_{1,1} \mid \mathbf{X}_{1}, \mathbf{X}_{2}\right]-\mathrm{E}\left[S_{1,1} \mid \mathbf{X}_{1}, Y_{2}\right]\right)\right\|^{2}\right]
\end{aligned}
$$


Moreover

$$
\begin{aligned}
\mathrm{E}[ & \left.S_{1,1} \mid \mathbf{X}_{1}, Y_{2}\right] \\
& \stackrel{(a)}{=} \mathrm{E}\left[\mathrm{E}\left[S_{1,1} \mid \mathbf{X}_{1}, \mathbf{X}_{2}\right] \mid \mathbf{X}_{1}, Y_{2}\right] \\
& \stackrel{(b)}{=} \mathrm{E}\left[\mathrm{E}\left[S_{1,1} \mid \mathbf{X}_{1}\right] \mid \mathbf{X}_{1}, Y_{2}\right]+\mathrm{E}\left[\mathrm{E}\left[S_{1,1} \mid \overline{\mathbf{X}}_{2}\right] \mid \mathbf{X}_{1}, Y_{2}\right] \\
& \stackrel{(c)}{=} \mathrm{E}\left[S_{1,1} \mid \mathbf{X}_{1}\right]+\mathrm{E}\left[\mathcal{W} \overline{\mathbf{X}}_{2} \mid \mathbf{X}_{1}, Y_{2}\right]
\end{aligned}
$$

where (a) follows from the successive conditioning property of conditional expectation and the fact that $Y_{2}$ is a function of $\mathbf{X}_{2}$, (b) from the fact that $E\left[S_{1,1} \mid \mathbf{X}_{1}, \mathbf{X}_{2}\right]=$ $E\left[S_{1,1} \mid \mathbf{X}_{1}\right]+E\left[S_{1,1} \mid \overline{\mathbf{X}}_{2}\right]$ in the jointly Gaussian case and from the linearity of conditional expectation and (c) from the successive conditioning property of conditional expectation and our jointly Gaussian assumption. The second term in the distortion (18) can thus be written as

$$
\begin{gathered}
\mathrm{E}\left[\left\|\mathcal{A}\left(\mathrm{E}\left[S_{1,1} \mid \mathbf{X}_{1}, \mathbf{X}_{2}\right]-\mathrm{E}\left[S_{1,1} \mid \mathbf{X}_{1}, Y_{2}\right]\right)\right\|^{2}\right] \\
\quad=\mathrm{E}\left[\left\|\mathcal{A}\left(\mathcal{W} \overline{\mathbf{X}}_{2}-\mathrm{E}\left[\mathcal{W} \overline{\mathbf{X}}_{2} \mid \mathbf{X}_{1}, Y_{2}\right]\right)\right\|^{2}\right] \\
\quad=\mathrm{E}\left[\left\|\mathcal{A}\left(\mathcal{W} \mathbf{X}_{2}-\mathrm{E}\left[\mathcal{W} \mathbf{X}_{2} \mid \mathbf{X}_{1}, Y_{2}\right]\right)\right\|^{2}\right] .
\end{gathered}
$$

It corresponds to the distortion of the innovation process $P_{1}$ and also follows from [40]. Using (18), the achieved distortion directly evaluates as $D_{1}(\theta)$, demonstrating the optimality of the encoding architecture depicted in Fig. 4.

\section{APPENDIX B}

\section{PROOF OF PROPOSITION 3.2}

The optimal encoding strategy reduces to that of a remote source coding problem. The rate $R_{1}(\theta)$ hence directly follows from the remote rate-distortion function in [31, Sec. 4.5.4]. The signal reconstructed at the decoder Dec 0 (see Fig. 3(b)), denoted $\hat{S}_{2,1}$, can thus be described by the system depicted in Fig. 16. The observed process $\mathbf{X}_{2}$ is first passed through a linear and time-invariant filter $\mathbf{g}_{1}$ with transfer function

$$
\mathbf{G}_{1}=\boldsymbol{\Phi}_{S_{21} X_{2}} \boldsymbol{\Phi}_{X_{2}}^{-1}
$$

to obtain the process $P_{1}$ whose PSD is given by

$$
\Phi_{P_{1}}=\Phi_{S_{21}}-\Phi_{S_{21} \mid X_{2}}=\boldsymbol{\Phi}_{S_{21} X_{2}} \boldsymbol{\Phi}_{X_{2}}^{-1} \boldsymbol{\Phi}_{S_{21} X_{2}}^{*} .
$$

We then add an independent Gaussian noise $Z_{1}$ with mean zero and PSD

$$
\Phi_{Z_{1}}=\max \left\{0, \frac{\theta \Phi_{P_{1}}}{|A|^{2} \Phi_{P_{1}}-\theta}\right\}
$$

for some $\theta \in\left(0, \operatorname{ess}_{\sup _{\Omega}}|A|^{2} \Phi_{P_{1}}\right]$. Finally, the resulting spectrum is band-limited by the filter $h_{1}$ whose frequency response is

$$
H_{1}=\max \left\{0, \frac{|A|^{2} \Phi_{P_{1}}-\theta}{|A|^{2} \Phi_{P_{1}}}\right\} .
$$

The filter $h_{1}$ is tantamount to an ideal band-limiting filter with transfer function $1_{\left\{|A|^{2} \Phi_{P_{1}} \geq \theta\right\}}$ whose output, denoted $M$, is then passed through the Wiener filter implementing the optimum MSE decoding rule $\hat{S}_{2,1}=\mathrm{E}\left[S_{2,1} \mid M\right]$. Since $\mathbf{X}_{1}$ is

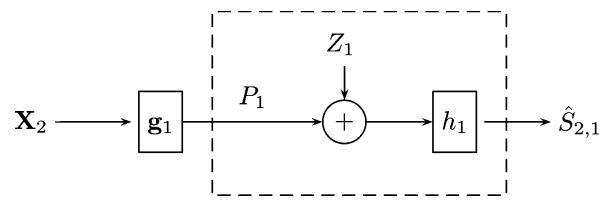

Fig. 16. Optimal reconstruction in the remote source coding problem. The dashed rectangle indicates the corresponding forward test channel.

available at the decoder Dec 1, and that we are interested in $S_{1,1}$ and not in $S_{2,1}$, the optimum MSE estimation rule may be replaced by $\hat{S}_{1,1}=\mathrm{E}\left[S_{1,1} \mid M, \mathbf{X}_{1}\right]$. The resulting weighted mean-squared reconstruction error can be expressed as

$$
\begin{aligned}
\frac{1}{2 \pi} & \int_{-\infty}^{\infty}|A|^{2} \Phi_{S_{11} \mid M, X_{1}} d \Omega \\
= & \frac{1}{2 \pi} \int_{-\infty}^{\infty}|A|^{2} \Phi_{S_{11} \mid M} d \Omega \\
& \quad-\frac{1}{2 \pi} \int_{-\infty}^{\infty}|A|^{2} \boldsymbol{\Phi}_{S_{11} \bar{X}_{1}} \boldsymbol{\Phi}_{\bar{X}_{1}}^{-1} \Phi_{S_{1} \bar{X}_{1}}^{*} d \Omega
\end{aligned}
$$

where $\overline{\mathbf{X}}_{1}=\mathbf{X}_{1}-\mathrm{E}\left[\mathbf{X}_{1} \mid M\right]$. The first term in (19) corresponds to the error made in a remote setup where no side information is available. The second term is the gain provided by the availability of $\mathbf{X}_{1}$ for the reconstruction. Evaluating the power spectral densities involved in (19) yields the claimed distortion formula.

\section{ACKNOWLEDGMENT}

The authors would like to thank the three anonymous reviewers for their valuable and constructive comments.

\section{REFERENCES}

[1] D. H. Johnson and D. E. Dudgeon, Array Signal Processing: Concepts and Techniques. Englewood Cliffs, NJ: Prentice-Hall, 1993.

[2] H. L. Van Trees, Optimum Array Processing. Part IV of Detection, Estimation and Modulation Theory. New York, NY: Wiley, 2002.

[3] T. Flynn and R. Gray, "Encoding of correlated observations," IEEE Trans. Inf. Theory, vol. 33, no. 6, pp. 773-787, Nov. 1987.

[4] H. Yamamoto and K. Itoh, "Source coding theory for communication systems with a remote source," Trans. IECE Jpn., vol. E63, pp. 700-706, Oct. 1980.

[5] S. C. Draper, "Successive structuring of source coding algorithms data fusion, buffering and distribution in networks," Ph.D. dissertation, MIT, Cambridge, MA, 2002.

[6] H. S. Witsenhausen, "Indirect rate-distortion problems," IEEE Trans. Inf. Theory, vol. 26, pp. 518-521, Sep. 1980.

[7] R. L. Dobrushin and B. S. Tsybakov, "Information transmission additional noise," IEEE Trans. Inf. Theory, vol. 8, pp. 293-304, Sep. 1962.

[8] D. J. Sakrison, "Source encoding in the presence of random disturbance," IEEE Trans. Inf. Theory, vol. 14, pp. 165-167, 1968.

[9] J. K. Wolf and J. Ziv, "Transmission of noisy information to a receiver with minimum distortion," IEEE Trans. Inf. Theory, vol. 16, pp. 406-411, Jul. 1970.

[10] D. Rebollo-Monedero, S. Rane, and B. Girod, "Wyner-Ziv and transform coding of noisy sources at high rates," in Proc. Asilomar Conf. Signals, Systems, Computers, Nov. 2004, vol. 2, pp. 2084-2088.

[11] D. Rebollo-Monedero, S. Rane, A. Aaron, and B. Girod, "High-rate quantization and transform coding with side information at the decoder," EURASIP J. Signal Process. (Special Issue on Source Coding), vol. 86, pp. 3160-3179, Nov. 2006.

[12] B. Widrow and F.-L. Luo, "Microphone arrays for hearing aids: An overview," Speech Commun. (Special Issue on Speech for Hearing Aids), vol. 39, pp. 139-146, Jan. 2003. 
[13] H. Luts, J.-B. Maj, W. Soede, and J. Wouters, "Better speech perception in noise with an assistive multimicrophone array for hearing aids," Ear Hear., vol. 25, pp. 411-420, Oct. 2004.

[14] B. Widrow, "A microphone array for hearing aids," IEEE Circuits Syst. Mag., vol. 1, pp. 26-32, Feb. 2001.

[15] J. G. Desloge, W. M. Rabinowitz, and P. M. Zurek, "Microphone-array hearing aids with binaural output—Part I: Fixed-processing systems," IEEE Trans. Signal Audio Process., vol. 5, pp. 529-542, Nov. 1997.

[16] D. P. Welker, J. E. Greenberg, J. G. Desloge, and P. M. Zurek, "Microphone-array hearing aids with binaural output-Part II: A two-microphone adaptive system," IEEE Trans. Speech Audio Process., vol. 5, pp. 543-551, Nov. 1997.

[17] T. J. Klasen, M. Moonen, T. van den Bogaert, and J. Wouters, "Preservation of interaural time delay for binaural hearing aids through Wiener filtering based noise reduction," in Proc. IEEE Int. Conf. Acoustics, Speech, Signal Processing, Mar. 2005, vol. 3, pp. 29-32.

[18] S. Doclo, D. Rong, T. J. Klasen, J. Wouters, S. Haykin, and M. Moonen, "Extension of the multi-channel Wiener filter with ITD cues for noise reduction in binaural hearing aids," in Proc. Workshop Applications Signal Processing to Audio and Acoustics, Oct. 2005, pp. 70-73.

[19] V. Richards, B. Moore, and S. Launer, "Potential benefits of acrossaid communication for bilaterally aided people: Listening in a car," Int. J. Audiolog., vol. 45, pp. 182-189, Mar. 2006.

[20] M. A. Lehr and B. Widrow, "Directional hearing system," U.S. Patent 5793 875-A, Aug. 1998.

[21] I. Holube, W. Meyer, K. Husung, A. Gebert, and G. Sporer, "Hörgeratesystem mit zwei hörhilfegeräten sowie verfahren zum eines solchen hörgerätesystems," Eur. Patent EP 941 014-A2, Sep. 1999.

[22] H.-U. Roeck, N. Cadalli, and P. Baechler, "Method for producing control signals, method of controlling signal transfer and a hearing device," Eur. Patent EP 1326 478-A2, Jul. 2003.

[23] H.-U. Roeck, "Binaural Hearing Device and Method for controlling a hearing device system," U.S. Patent 2004175 005-A1, Sep. 2004.

[24] H. Baechler, "Binaural synchronization," U.S. Patent 2004208 332-A1, Oct. 2004.

[25] S. Doclo, T. van den Bogaert, M. Moonen, and J. Wouters, "Reducedbandwidth and distributed MWF-based noise reduction algorithms binaural hearing aids," in IEEE Trans. Audio, Speech, Lang. Process., 2008, accepted for publication.

[26] J. E. Greenberg, P. M. Peterson, and P. M. Zurek, "Intelligibilityweighted measures of speech-to-interference ratio and speech performance," J. Acoust. Soc. Amer., vol. 94, pp. 3009-3010, Feb. 1993.

[27] M. L. Hawley, R. Y. Litovsky, and J. F. Culling, "The benefit of binaural hearing in a cocktail party: Effect of location and type of interferer," J. Acoust. Soc. Amer., vol. 115, pp. 833-843, Feb. 2004.

[28] S. Doclo, T. J. Klasen, T. van den Bogaert, J. Wouters, and M. Moonen, "Theoretical analysis of binaural cue preservation using multi-channel wiener filtering and interaural transfer functions," presented at the Int. Workshop Acoustic Echo Noise Control, Paris, France, Sep. 2006.

[29] T. Klasen, T. Van den Bogaert, M. Moonen, and J. Wouters, "Binaural noise reduction algorithms for hearing aids that preserve interaural delay cues," IEEE Trans. Signal Process., vol. 55, no. 4, pp. 1579-1585, Apr. 2007.

[30] S. S. Pradhan and K. Ramchandran, "Distributed source coding using syndromes (DISCUS): Design and construction," IEEE Trans. Inf. Theory, vol. 49, pp. 626-634, Mar. 2003.

[31] T. Berger, Rate Distortion Theory: A Mathematical Basis for Data Compression. Englewood Cliffs, NJ: Prentice-Hall, 1971.

[32] A. D. Wyner and A. D. Ziv, "The rate-distortion function for source coding with side information at the decoder," IEEE Trans. Inf. Theory, vol. 22, no. 1, pp. 1-10, Jan. 1976.

[33] R. A. Horn and C. R. Johnson, Matrix Analysis. Cambridge, MA: Cambridge Univ. Press, 1985.

[34] S. Boyd and L. Vandenberghe, Convex Optimization. Cambridge, MA: Cambridge Univ. Press, 2004.

[35] M. Nilsson, S. D. Soli, and A. Sullivan, "Development of the hearing in noise test for the measurement of speech reception thresholds in quiet and in noise," J. Acoust. Soc. Amer., vol. 95, pp. 1085-1099, Feb. 1994.

[36] A. P. Varga, H. J. M. Steeneken, M. Tomlinson, and D. Jones, "The NOISEX-92 study on the effect of additive noise on automative speech recognition," Speech Research Unit, Defense Research Agency, Malvern, U.K., Tech. Rep., 1992.

[37] American National Standard methods for calculation of the Speech Intelligibility Index, ANSI S3.5-1997, 1997.

[38] T. M. Cover and J. A. Thomas, Elements of Information Theory. New York: Wiley, 1991.
[39] M. Gastpar, P. L. Dragotti, and M. Vetterli, "The distributed karhunenloève transform," IEEE Trans. Inf. Theory, pp. 5177-5196, Dec. 2006.

[40] D. J. Sakrison, "The rate distortion function of a Gaussian process a weighted square error criterion," IEEE Trans. Inf. Theory, vol. 14, pp. 506-508, May 1968.

[41] S. S. Pradhan and K. Ramchandran, "Enhancing analog image transmission systems using digital side information: A new wavelet-based image coding paradigm," in Proc. IEEE Data Compression Conf., Mar. 2001, pp. 63-72.

[42] U. Grenander and G. Szegö, Toeplitz. Forms and Their Applications, 2nd ed. New York: Chelsea, 1984.

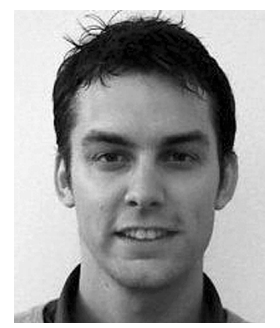

Olivier Roy (S'05) received the M.S. degree in communication systems in 2004, and the Ph.D. degree in signal processing in 2008 from the Ecole Polytechnique Fédérale de Lausanne (EPFL), Switzerland.

From March to September 2002, he was a Software Engineer at Logitech, Fremont, CA, where he worked on Internet video streaming. From August 2003 to March 2004, he was a research intern at the Institute for Telecommunications Research, University of South Australia, Mawson Lakes, Australia, where he investigated information-theoretic aspects of multiantenna communication systems. From June to August 2007, he was with Phonak, a hearing aid company based in Stäfa, Switzerland, where he developed noise reduction schemes for binaural hearing aids. Since 2004, he has been a Research Assistant at the Audiovisual Communications Laboratory at EPFL. His research interests are in distributed estimation and coding, microphone array processing and digital hearing aids.

Mr. Roy received a Best Student Paper Award at the Australian Communication Theory Workshop in 2004.

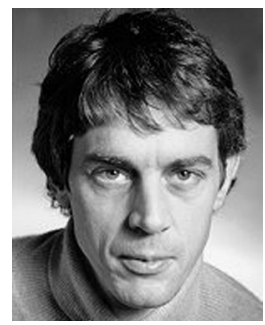

Martin Vetterli (F'95) received the Dipl.El.-Ing. degree from ETH Zurich (ETHZ), Switzerland, in 1981, the M.S. degree from Stanford University, Stanford, CA, in 1982, and the Doctoratès Sciences degree from EPF Lausanne (EPFL) in 1986.

He was a Research Assistant at Stanford and EPFL and has worked for Siemens and AT\&T Bell Laboratories. In 1986, he joined Columbia University, New York, where he was most recently an Associate Professor of electrical engineering and co-director of the Image and Advanced Television Laboratory. In 1993, he joined the University of California at Berkeley, where he was a Professor in the Department of Electrical Engineering and Computer Sciences until 1997, and now holds an Adjunct Professor position. Since 1995, he has been a Professor of Communication Systems at EPF Lausanne, Switzerland, where he chaired the Communications Systems Division from 1996 to 1997m, and heads the Audiovisual Communications Laboratory. From 2001 to 2004, he directed the National Competence Center in Research on mobile information and communication systems. He has also been a Vice-President for International Affairs at EPFL since October 2004. He has held visiting positions at ETHZ (1990) and Stanford University (1998). He is the coauthor, with J. Kovačević, of the book Wavelets and Subband Coding (Prentice-Hall, 1995). He has published about 85 journal papers on a variety of topics in signal/image processing and communications and holds seven patents. His research interests include sampling, wavelets, multirate signal processing, computational complexity, signal processing for communications, digital video processing, and joint source/channel coding.

Dr. Vetterli is a member of SIAM and was the Area Editor for Speech, Image, Video, and Signal Processing of the IEEE TRANSACTIONS ON COMMUNICATIONS. He is also on the editorial boards of Annals of Telecommunications, Applied and Computational Harmonic Analysis, and The Journal of Fourier Analysis and Application. He received the Best Paper Award of EURASIP in 1984 for his paper on multidimensional subband coding, the Research Prize of the Brown Bovery Corporation (Switzerland) in 1986 for his doctoral thesis, the IEEE Signal Processing Society's Senior Awards in 1991 and 1996 (for papers with D. LeGall and K. Ramchandran, respectively). He won the Swiss National Latsis Prize in 1996, the SPIE Presidential award in 1999, and the IEEE Signal Processing Technical Achievement Award in 2001. He was a member of the Swiss Council on Science and Technology until December 2003. He was a plenary speaker at various conferences (e.g., 1992 IEEE ICASSP). 